\title{
CONNECTEDNESS IN FINE TOPOLOGIES
}

\author{
J. Heinonen, T. Kilpeläinen, and J. Malý
}

\section{Introduction}

If $E$ is an arbitrary subset of Euclidean $n$-space $\mathbf{R}^{n}$, let $B_{\alpha, p}(E)$ denote the Bessel capacity of $E, 0<\alpha<\infty, 1<p \leq n / \alpha$, that is

$$
B_{\alpha, p}(E)=\inf \left\{\|f\|_{p}^{p}: f \in L_{+}^{p}\left(\mathbf{R}^{n}\right), G_{\alpha} * f \geq 1 \text { in } E\right\} .
$$

Here $L^{p}\left(\mathbf{R}^{n}\right)$ is the usual Lebesgue space of $p$-th power summable functions, $L_{+}^{p}\left(\mathbf{R}^{n}\right)$ the nonnegative elements, $\|f\|_{p}$ the usual norm of $f$ in $L_{p}$, and $G_{\alpha} * f$ the convolution over $\mathbf{R}^{n}$ of $f$ with the Bessel kernel $G_{\alpha}$, best defined by its Fourier transform $\hat{G}_{\alpha}(\xi)=\left(1+|\xi|^{2}\right)^{-\alpha / 2}$, see e.g. [St]. The reader should note that the Bessel capacity is a Choquet capacity.

As usually in nonlinear potential theories, we say that the set $E$ is $(\alpha, p)$-thin at $x$ in $\mathbf{R}^{n}$ if the Wiener integral converges,

$$
\int_{0}^{1}\left(r^{\alpha p-n} B_{\alpha, p}(E \cap B(x, r))\right)^{1 /(p-1)} \frac{d r}{r}<\infty .
$$

Here $B(x, r)$ is the open ball $\left\{y \in \mathbf{R}^{n}:|x-y|<r\right\}$. If $E$ is not $(\alpha, p)$-thin at $x$, then we say that $E$ is $(\alpha, p)$-fat at $x$. The set $b(E)$ of points at which $E$ is $(\alpha, p)$-fat is called the $(\alpha, p)$-base of $E$.

We define the $(\alpha, p)$-fine topology, $\tau_{\alpha, p}$, to be the collection of all sets $V \subset \mathbf{R}^{n}$ such that $V^{c}$, the complement of $V$, is $(\alpha, p)$-thin at each $x \in V$. Thus $V$ is an $(\alpha, p)$-fine neighborhood of $x \in V$ if and only if $V^{c}$ is $(\alpha, p)$-thin at $x$, cf. [M2, p. 162]. Topological concepts in $(\alpha, p)$-fine topology are equipped with the phrase " $(\alpha, p)$-fine", for example $(\alpha, p)$-finely open, $(\alpha, p)$-finely connected, or if no confusion arises, finely open, finely compact, etc.

The particular case of $(\alpha, p)$-fine topologies when $\alpha=1$ and $1<p \leq n$ is related to second order elliptic equations. As well known, the $(1,2)$-fine topology coincides with the classical fine topology of $\mathrm{H}$. Cartan, the coarsest topology on

Part of this work was done in Spring 1988 when all the authors were at the University of Jyväskylä. The third author takes this opportunity to express his gratitude to the Mathematics Department in Jyväskylä for its hospitality.

doi:10.5186/aasfm.1990.1514 
$\mathbf{R}^{n}$ making all superharmonic functions continuous. A similar result holds for all $p$ not greater than $n$ : the $(1, p)$-fine topology is the coarsest topology in which all supersolutions of the $p$-Laplace equation

$$
\operatorname{div}\left(|\nabla u|^{p-2} \nabla u\right)=0
$$

are continuous. In effect, the equation (1.2) can be replaced by a more general degenerate elliptic equation

$$
\operatorname{div} \mathcal{A}(x, \nabla u)=0
$$

where $\mathcal{A}(x, h) \cdot h \approx|h|^{p}$. See $[\mathrm{HKM}]$ and Section 6 below.

There are several sources for the various properties of the Bessel capacities, the associated nonlinear potentials, and the use of the $(\alpha, p)$-fine topologies in analysis. We refer the reader e.g. to $[\mathrm{AH}],[\mathrm{AL}],[\mathrm{AM}]$, [Hed], [HW], [M1-2], [MK], and [R2]. See also [F1]. However, topological properties of $(\alpha, p)$-fine topologies are not yet thoroughly investigated. In [AL] D.R. Adams and J.L. Lewis showed that for $\alpha p>1$ each $(\alpha, p)$-finely open and $(\alpha, p)$-finely connected set is arcwise connected; the result is false if $\alpha p \leq 1$. Our main results in this paper assert that the $(\alpha, p)$-fine topology is locally connected (provided $\alpha p>1$ ) and it obeys Doob's quasi-Lindelöf principle: any collection of $(\alpha, p)$-finely open sets has a countable subcollection whose union differs from the union of the whole family only by a set of $(\alpha, p)$-capacity zero. See Sections 2 and 3. In classical potential theory these two properties are proved using the balayage of measures, a tool which is not available in this nonlinear setting. In the linear situation these results are found in [F2] and [D].

We will also show that if $\alpha p>n-1$, then an $(\alpha, p)$-finely open set is finely connected, arcwise connected and euclidean connected at the same time, the assertion being false if $\alpha p \leq n-1$. See Section 5. The case $\alpha p>n-1$ thus resembles the classical plane case where this result is known [F3], [GL].

In the final section, Section 6 , we apply the aforementioned arcwise connectedness result for asymptotic paths of $\mathcal{A}$-subharmonic functions.

\section{The quasi-Lindelöf property}

In this section we show that the $(\alpha, p)$-fine topologies obey Doob's quasiLindelöf principle.

First we prove an auxiliary result, a modified Wiener criterion.

2.1. Lemma. Let $x_{0} \in \mathbf{R}^{n}$. Suppose that $x_{k}$ is a sequence of points with $\left|x_{k}-x_{0}\right|<2^{-k-2}, k=1,2, \ldots$ If $B_{k}\left(x_{k}\right)=B\left(x_{k}, 3 \cdot 2^{-k-2}\right)$, then a set $E \subset \mathbf{R}^{n}$ is $(\alpha, p)$-thin at $x_{0}$ if and only if

$$
\sum_{k=1}^{\infty}\left(2^{-k(\alpha p-n)} B_{\alpha, p}\left(E \cap B_{k}\left(x_{k}\right)\right)\right)^{1 /(p-1)}<\infty .
$$


Proof. Since

$$
B\left(x_{0}, 2^{-k-1}\right) \subset B_{k}\left(x_{k}\right) \subset B\left(x_{0}, 2^{-k}\right),
$$

the claim follows easily.

The Kellogg property was proved in [HW, Theorem 2]:

2.2. The Kellogg property. Let $E$ be any set in $\mathbf{R}^{n}$. If $e_{\alpha, p}(E)$ is the set of all points at which $E$ is $(\alpha, p)$-thin, then

$$
B_{\alpha, p}\left(e_{\alpha, p}(E) \cap E\right)=0 .
$$

To state our main result in this section recall that $\tau_{\alpha, p}$ has the quasi-Lindelof property if for each family $\left\{U_{\lambda}\right\}, \lambda \in \Lambda$, of $(\alpha, p)$-finely open sets there is a countable set $\Gamma \subset \Lambda$ such that

$$
B_{\alpha, p}\left(\bigcup_{\lambda \in \Lambda} U_{\lambda} \backslash \bigcup_{\lambda \in \Gamma} U_{\lambda}\right)=0
$$

We prove

2.3. Theorem. The $(\alpha, p)$-fine topology $\tau_{\alpha, p}$ has the quasi-Lindelöf property.

Proof. We make use of the following local capacity: Let $\left\{B_{k}\right\}, k=1,2, \ldots$, be the collection of all balls $B \subset \mathbf{R}^{n}$ with rational centers and radii. Write

$$
\operatorname{cap}(E)=\sum_{k=1}^{\infty} 2^{-k} \frac{B_{\alpha, p}\left(E \cap B_{k}\right)}{B_{\alpha, p}\left(B_{k}\right)}
$$

for $E \subset \mathbf{R}^{n}$. Then, clearly, $\operatorname{cap}(\cdot)$ is a subadditive set function and $B_{\alpha, p}(E)=0$ if and only if $\operatorname{cap}(E)=0$.

Suppose then that the sets $U_{\lambda}, \lambda \in \Lambda$, are $(\alpha, p)$-finely open and that

$$
U=\bigcup_{\lambda \in \Lambda} U_{\lambda}
$$

Let

$$
\delta=\inf \left\{\operatorname{cap}\left(U \backslash \bigcup_{\lambda \in \Gamma} U_{\lambda}\right): \Gamma \subset \Lambda \text { countable }\right\}
$$

Then choosing countable sets $\Gamma_{j} \subset \Lambda, j=1,2, \ldots$, with

$$
\operatorname{cap}\left(U \backslash \bigcup_{\lambda \in \Gamma_{j}} U_{\lambda}\right) \leq \delta+1 / j
$$


and putting

$$
\Gamma_{0}=\bigcup_{j=1}^{\infty} \Gamma_{j}
$$

we obtain

$$
\delta=\operatorname{cap}(F)
$$

where

$$
F=U \backslash \bigcup_{\lambda \in \Gamma_{0}} U_{\lambda}
$$

To complete the proof we show that $\delta=0$. Suppose, on the contrary, that $\delta>0$. Since $B_{\alpha, p}(F)>0$, it follows from the Kellogg property that there is a point $x \in F \cap b(F) \subset U$. Then choose $\lambda \in \Lambda$ such that $x \in U_{\lambda}$. Now, since $F \backslash U_{\lambda}$ is $(\alpha, p)$-thin and $F(\alpha, p)$-fat at $x$, Lemma 2.1 enables us to pick a ball $B_{k}$ with rational center and radius such that

$$
B_{\alpha, p}\left(\left(F \backslash U_{\lambda}\right) \cap B_{k}\right)<B_{\alpha, p}\left(F \cap B_{k}\right)
$$

whence

$$
\operatorname{cap}\left(F \backslash U_{\lambda}\right)<\operatorname{cap}(F)=\delta
$$

which is a contradiction. The theorem follows.

\section{Local connectedness}

This section is devoted to proving that the $(\alpha, p)$-fine topology is locally connected provided $\alpha p>1$. This is not true if $\alpha p \leq 1$.

Recall that the $(\alpha, p)$-base of the set $E$ is

$$
b(E)=\left\{x \in \mathbf{R}^{n}: E \quad \text { is }(\alpha, p) \text {-fat at } x\right\} .
$$

We have

3.1. Proposition. The $(\alpha, p)$-base $b(E)$ of a set $E \subset \mathbf{R}^{n}$ is a $G_{\delta}$-set.

Proof. The sets

$$
G_{k}=\left\{x \in \mathbf{R}^{n}: \int_{0}^{1}\left(r^{\alpha p-n} B_{\alpha, p}(E \cap B(x, r))\right)^{1 /(p-1)} \frac{d r}{r}>k\right\}
$$

are easily seen to be open and, clearly,

$$
b(E)=\bigcap_{k=1}^{\infty} G_{k} .
$$

The lemma follows.

We call a set $U \subset \mathbf{R}^{n}(\alpha, p)$-finely regular if $U^{c}=b\left(U^{c}\right)$. Proposition 3.1 immediately yields 
3.2. Corollary. An $(\alpha, p)$-finely regular set $U$ is $(\alpha, p)$-finely open and of type $F_{\sigma}$.

3.3. Remark. It is easily seen that the $(\alpha, p)$-fine interior of any $(\alpha, p)$-finely closed set is $(\alpha, p)$-finely regular.

Next we establish the Lusin-Menchoff property (or binormality) for the $(\alpha, p)$ fine topology.

3.4. Theorem. Suppose that $F \subset \mathbf{R}^{n}$ is (euclidean) compact and $T \subset \mathbf{R}^{n}$ $(\alpha, p)$-finely closed with $T \cap F=\emptyset$. Then there is an open set $G \subset \mathbf{R}^{n}$ such that

$$
T \subset G \subset G \cup b(G) \subset F^{c} \text {. }
$$

Proof. We proceed with the proof inductively. Put $T_{0}=T$ and let $k \in \mathbf{N}$. Then choose a finite set $\mathcal{Z}_{k} \subset F$ such that

$$
F \subset \bigcup_{z \in \mathcal{Z}_{k}} B\left(z, 2^{-k-2}\right) \text {. }
$$

Write

$$
P_{k}=\left\{x \in \mathbf{R}^{n}: \operatorname{dist}(x, F) \leq 3 \cdot 2^{-k-3}\right\} .
$$

For each $j=1,2, \ldots, k$ and $z \in \mathcal{Z}_{j}$ choose an open neighborhood $G_{k, j, z}$ of $T_{k-1}$ such that

$$
B_{\alpha, p}\left(G_{k, j, z} \cap B_{j}(z)\right) \leq\left(2-2^{-k}\right) B_{\alpha, p}\left(T \cap B_{j}(z)\right)
$$

where $B_{j}(z)=B\left(z, 3 \cdot 2^{-j-2}\right)$. Then putting

$$
T_{k}=T_{k-1} \cup\left(\bigcap_{j, z} G_{k, j, z} \backslash P_{k}\right)
$$

we obtain

$$
B_{\alpha, p}\left(T_{k} \cap B_{j}(z)\right) \leq\left(2-2^{-k}\right) B_{\alpha, p}\left(T \cap B_{j}(z)\right)
$$

for every $j=1,2, \ldots, k+1$ and $z \in \mathcal{Z}_{j}$ since

$$
T_{k} \cap B_{k+1}(z) \subset T_{k} \cap P_{k} \subset T .
$$

To complete the proof write

$$
G=\bigcup_{k=1}^{\infty} T_{k}
$$

Clearly, $G$ is open and $T \subset G \subset F^{c}$. Moreover, it follows from (3.5) that

$$
B_{\alpha, p}\left(G \cap B_{j}(z)\right) \leq 2 B_{\alpha, p}\left(T \cap B_{j}(z)\right)
$$

for all $j \in \mathbf{N}$ and $z \in \mathcal{Z}_{j}$. Hence Lemma 2.1 implies that

$$
b(G) \cap F=\emptyset
$$

and the theorem is proved. 
3.6. Remarks. (a) Stated differently, Theorem 3.4 says that $F$ has an $(\alpha, p)$-finely open neighborhood $U$ and $T$ an open neighborhood $G$ such that $U \cap G=\emptyset$.

(b) Theorem 3.4 holds also if $F$ is assumed to be closed instead of compact, see Corollary 3.8 below and the proof of [LMZ, 10.25].

The next two corollaries follow using [LMZ, 3.13 and 3.14].

3.7. Corollary. The $(\alpha, p)$-fine topology is completely regular.

3.8. Corollary. If $U$ is an $(\alpha, p)$-finely open $F_{\sigma}$-set, then there is an upper semicontinuous and $(\alpha, p)$-finely continuous function $f: \mathbf{R}^{n} \rightarrow[0,1]$ such that

$$
U=\left\{x \in \mathbf{R}^{n}: f(x)>0\right\} .
$$
$3.16]$.

3.9. Remarks. (a) It also follows that $\left(\mathbf{R}^{n}, \tau_{\alpha, p}\right)$ is a Baire space; see [LMZ,

(b) Corollary 3.7 implies that $(\alpha, p)$-regular open sets form a base for the $(\alpha, p)$-fine topology $\tau_{\alpha, p}$, cf. Remark 3.3.

We say that a property holds $(\alpha, p)$-quasieverywhere, abbreviated $(\alpha, p)$-q.e., if it holds except on a set of $(\alpha, p)$ - capacity zero. A function $f$, defined $(\alpha, p)$-q.e., is called $(\alpha, p)$-quasicontinuous if for every $\varepsilon>0$ there is an open set $G$ such that $B_{\alpha, p}(G)<\varepsilon$ and that the restriction $\left.f\right|_{G^{c}}$ is continuous. Then a function is $(\alpha, p)$-quasicontinuous if and only if it is $(\alpha, p)$-finely continuous $(\alpha, p)$-q.e. [HW, Theorem 8].

3.10. Theorem. Suppose that $f$ is an $(\alpha, p)$-finely continuous function on $\mathbf{R}^{n}$. Then each $x_{0} \in \mathbf{R}^{n}$ has an $(\alpha, p)$-fine neighborhood $W$ of $x_{0}$ such that $\left.f\right|_{W}$ is continuous.

Proof. Similarly to [HKM, 3.17] one can easily show that there is a set $E \subset \mathbf{R}^{n}$ which is $(\alpha, p)$-thin at $x_{0}$ such that

$$
\lim _{\substack{x \rightarrow x_{0} \\ x \notin E}} f(x)=f\left(x_{0}\right)
$$

Fix $j \in \mathbf{N}$. Since $f$ is quasicontinuous, there is an open set $G_{j} \subset B\left(x_{0}, 2^{-j+2}\right) \backslash$ $B\left(x_{0}, 2^{-j-1}\right)$ such that the restriction

$$
\left.f\right|_{\bar{B}\left(x_{0}, 2^{-j+1}\right) \backslash\left(B\left(x_{0}, 2^{-j}\right) \cup G_{j}\right)}
$$

is continuous and

$$
B_{\alpha, p}\left(G_{j}\right)<2^{-2 j n}
$$


Writing

$$
A=\bigcup_{j=1}^{\infty} G_{j} \cup E
$$

and $W=B\left(x_{0}, 1\right) \backslash A,(3.11)$ implies that $W$ is an $(\alpha, p)$-fine neighborhood of $x_{0}$. Moreover, $\left.f\right|_{W}$ is continuous as desired.

For the next lemma we need to assume that $\alpha p>1$.

3.12. Lemma. Suppose that $\alpha p>1$ and that $U$ is an $(\alpha, p)$-fine neighborhood of $x_{0}$. Then there is an $(\alpha, p)$-fine neighborhood $V$ of $x_{0}, V \subset U$, such that $V$ is connected in the euclidean topology.

Proof. By [AL, Theorem 2] there is an $(\alpha, p)$-fine neighborhood $V^{\prime}$ of $x_{0}$, $V^{\prime} \subset U$, such that for each $x \in V^{\prime}$ there is an arc $\gamma_{x}$ joining $x$ to $x_{0}$ in $U$. Then

$$
V=\bigcup_{x \in V^{\prime}} \gamma_{x}
$$

is the desired $(\alpha, p)$-fine neighborhood of $x_{0}$.

3.13. Remark. Lemma 3.12 is false if $\alpha p \leq 1$. Indeed, let

$$
U=\left(\bigcup_{j=1}^{\infty} \partial B(0,1 / j)\right)^{c} .
$$

Since $B_{\alpha, p}(\partial B(0,1 / j))=0$ for $\alpha p \leq 1$ [M1, Theorem 21], $U$ is $(\alpha, p)$-finely open. On the other hand, $\{0\}$ is the euclidean 0 -component of $U$. Hence Lemma 3.12 fails to hold if $\alpha p \leq 1$.

3.14. Lemma. Suppose that $\alpha p>1$ and that $U$ is an $(\alpha, p)$-finely regular set. Then the family

$$
C l o p U=\{V \subset U: V \text { and } U \backslash V \text { are }(\alpha, p) \text {-finely open }\}
$$

is a $\sigma$-algebra on $U$.

Proof. Let $V_{k} \in C \operatorname{lop} U, k=1,2, \ldots$, and $x_{0} \in \cap_{k=1}^{\infty} V_{k}$. It suffices to show that $\cap V_{k}$ is an $(\alpha, p)$-fine neighborhood of $x_{0}$. Since for each integer $k$, the sets $V_{k}$ and $U \backslash V_{k}$ are $(\alpha, p)$-finely regular, Corollaries 3.2 and 3.8 allow us to choose upper semicontinuous and $(\alpha, p)$ - continuous functions $f_{k}$ and $g_{k}, 0 \leq f_{k}, g_{k} \leq 1$, such that

$$
V_{k}=\left\{x \in \mathbf{R}^{n}: f_{k}(x)>0\right\}
$$

and

$$
U \backslash V_{k}=\left\{x \in \mathbf{R}^{n}: g_{k}(x)>0\right\}
$$


Write

$$
f=\sum_{k=1}^{\infty} 2^{-k}\left(f_{k}+g_{k}\right)
$$

Then $f$ is $(\alpha, p)$-finely continuous whence, by Theorem 3.10 , there is an $(\alpha, p)$-fine neighborhood $\tilde{W} \subset U$ of $x_{0}$ such that $\left.f\right|_{\tilde{W}}$ is (euclidean) continuous. In light of Lemma 3.12 we may pick an $(\alpha, p)$-fine neighborhood $W$ of $x_{0}, W \subset \tilde{W}$, such that $W$ is connected. Then fix $k \in \mathbf{N}$. Since $\left.f\right|_{W}$ is continuous and $f_{k}$ and $g_{k}$ are upper semicontinuous, the restrictions $\left.f_{k}\right|_{W}$ and $\left.g_{k}\right|_{W}$ are continuous. Thus the sets $V_{k} \cap W$ and $\left(U \backslash V_{k}\right) \cap W$ are relatively open in $W$. Since $W$ is connected and $x_{0} \in V_{k} \cap W$ it follows that $W \subset V_{k}$. Consequently,

$$
W \subset \bigcap_{k=1}^{\infty} V_{k}
$$

whence $\cap_{k=1}^{\infty} V_{k}$ is an $(\alpha, p)$-fine neighborhood of $x_{0}$ as desired.

Now we are ready to prove our principal theorem. nected.

3.15. Theorem. If $\alpha p>1$, then the $(\alpha, p)$-fine topology is locally con-

Proof. Let $U_{0}$ be an $(\alpha, p)$-fine neighborhood of $x_{0}$. Choose an $(\alpha, p)$-finely regular neighborhood $U$ of $x_{0}$ such that $U \subset U_{0}$. Write

$$
\begin{aligned}
C l o p_{x_{0}} U=\{V \subset U: & x_{0} \in V, V \text { is }(\alpha, p) \text {-finely open } \\
& \text { and } U \backslash V \text { is }(\alpha, p) \text {-finely open }\} .
\end{aligned}
$$

Using the quasi-Lindelöf property (Theorem 2.3) we find a sequence $V_{k} \in C l o p_{x_{0}} U$ such that the set

$$
F=\bigcup\left\{U \backslash V: V \in C l o p_{x_{0}} U\right\} \backslash \bigcup_{k=1}^{\infty}\left(U \backslash V_{k}\right)
$$

has the $(\alpha, p)$-capacity zero. Then Lemma 3.14 implies that

$$
W=\bigcap_{k=1}^{\infty} V_{k} \backslash F
$$

is an $(\alpha, p)$-fine neighborhood of $x_{0}$. On the other hand, $W$ is the $(\alpha, p)$-fine component containing $x_{0}$ since

$$
W=\bigcap\left\{V: V \in C l o p_{x_{0}} U\right\}
$$

The proof is complete.

3.16. Remark. The example in Remark 3.13 shows that the $(\alpha, p)$-fine topology is not locally connected if $\alpha p \leq 1$. 


\section{Variational capacity and Hausdorff measures}

Throughout this section let $\alpha=m$ be a positive integer and $p>1$ such that $m p \leq n$. We present some results concerning capacity and measure densities; these results, mostly known, will be needed in Section 5 .

Let $U$ and $\Omega$ be open sets in $\mathbf{R}^{n}$ with $U \subset \subset \Omega$. Define the variational $(m, p)$-capacity of $U$ in $\Omega$ to be the number

$$
\operatorname{cap}_{m, p}(U, \Omega)=\inf \sum_{|\alpha|=m} \int_{\Omega}\left|D^{\alpha} \varphi\right|^{p} d x
$$

where the infimum is taken over all $\varphi \in C_{0}^{\infty}(\Omega)$ with $\varphi \geq 1$ in $U$. If $E \subset \subset \Omega$ is any set we define

$$
\operatorname{cap}_{m, p}(E, \Omega)=\inf _{\substack{E \subset U \\ U \subset \subset \Omega \text { open }}} \operatorname{cap}_{m, p}(U, \Omega)
$$

Then there are constants $c_{1}=c_{1}(n, m, p, \operatorname{dist}(E, \partial \Omega))$ and $c_{2}=c_{2}(n, m, p$, $\operatorname{diam}(\Omega))$ such that

$$
c_{1} B_{m, p}(E) \leq \operatorname{cap}_{m, p}(E, \Omega) \leq c_{2} B_{m, p}(E)
$$

see $[R 2$, Section 6].

Let $h:[0, \infty) \rightarrow[0, \infty)$ be a continuous nondecreasing function with $h(0)=0$ and $\lim _{r \rightarrow \infty} h(r)=\infty$. We define the h-Hausdorff measure (or content) of a set $E$ by

$$
H_{h}(E)=\inf \left\{\sum_{i} h\left(r_{i}\right): E \subset \bigcup_{i=1}^{\infty} B\left(x_{i}, r_{i}\right)\right\} .
$$

The following theorem is due to Yu.G. Reshetnyak [R1, Lemma 6] and [R2, Theorem 4.1]; see also [Mar]. We briefly indicate how to deduce it from Reshetnyak's results.

4.1. Theorem. Suppose that

$$
\int_{0}^{2 r}\left(\frac{h(t)}{t^{n-m p}}\right)^{1 / p} \frac{d t}{t}=I(r)<\infty
$$

for all $0<r \leq r_{0} \leq 1$. Then for each open set $E \subset \mathbf{R}^{n}$ and $x \in \mathbf{R}^{n}$

$$
H_{h}(E \cap B(x, r)) \leq c I(r)^{p} \operatorname{cap}_{m, p}(E \cap B(x, r), B(x, 2 r))
$$

for $r \leq r_{0}$. Here $c=c(n, m, p)$. 
The proof is based on the following lemma [R1, Lemma 6].

4.2. Lemma. If $u \in L^{p}(B(2 r))$ is nonnegative, sptu $\subset B(2 r)$ and

$$
v(x)=\int_{B(2 r)} \frac{u(y)}{|x-y|^{n-m}} d y,
$$

then there are constants $K_{1}=K_{1}(n, m, p), K_{2}=K_{2}(n, p)$ and $K_{3}=K_{3}(n)$ such that

$$
H_{h}\left(\left\{x: v(x)>\frac{K_{1} I(r)}{\delta}+K_{2} r^{m-n / p}\|u\|_{p}\right\}\right) \leq K_{3} \delta^{p}\|u\|_{p}^{p}
$$

for all $\delta>0$.

Proof of Theorem 4.1. Fix $r \leq r_{0}$. Choose $\varphi \in C_{0}^{\infty}(B(2 r))$ such that $\varphi=1$ in $E \cap B(r)$. Then, by [R2, Lemma 6.2],

$$
\varphi(x) \leq c_{1}(n, m) \int_{B(2 r)} \sum_{|\alpha|=m} \frac{\left|D^{\alpha} \varphi(y)\right|}{|x-y|^{n-m}} d y .
$$

Write

$$
u(y)=c_{1} \sum_{|\alpha|=m}\left|D^{\alpha} \varphi(y)\right| .
$$

Since there is a constant $c=c(n, m)>0$ such that

$$
c^{-1} \inf \int_{B(2 r)}|u|^{p} d m \leq \operatorname{cap}_{m, p}(E \cap B(r), B(2 r)) \leq c \inf \int_{B(2 r)}|u|^{p} d m
$$

where the infimum is taken over all such $u$, we may assume that

$$
\|u\|_{p}<\frac{1}{2} K_{2}^{-1} r^{(n-m p) / p}
$$

where $K_{2}$ is the constant of Lemma 4.2.

Now choosing $\delta=2 K_{1} I(r)$ Lemma 4.2 yields

$$
H_{h}(E \cap B(r)) \leq H_{h}(\{x: \varphi(x) \geq 1\}) \leq c(n, m, p) I(r)^{p}\|u\|_{p}^{p} .
$$

This completes the proof.

4.3. Remark. As well known, the converse inequality for Theorem 4.1 holds with the function $h(r)=r^{n-m p}$ if $m p<n, h(r)=(\log (2 / r))^{1-p}$ if $m p=n$, see e.g. [MK], [R2, Theorem 4.2] and [Mar, 4.1]. A survey of comparison theorems can be found in [Hed].

4.4. Remark. If $m p<n$ it follows from the Sobolev embedding theorem that for $r \leq 1$

$$
\operatorname{cap}_{m, p}(E, B(2)) \leq \operatorname{cap}_{m, p}(E, B(2 r)) \leq c \operatorname{cap}_{m, p}(E, B(2))
$$

whenever $E \subset B(r)$. Here $c=c(n, m, p)$, cf. [Maz, Proposition 9.1.1/3]. Hence

$$
c_{1} B_{m, p}(E) \leq \operatorname{cap}_{m, p}(E, B(2 r)) \leq c_{2} B_{m, p}(E)
$$

whenever $E \subset B(r)$. Here $c_{i}=c_{i}(n, m, p), i=1,2$.

If $m p=n$, the assertions (4.5) and (4.6) do not hold. 


\section{Comparison between different types of connectedness}

Our main result in this section reads: for $\alpha p>n-1$ the $(\alpha, p)$-finely open set is $(\alpha, p)$-finely connected, arcwise connected, and (euclidean) connected at the same time. rem B].

We start with two auxiliary results. The first is a consequence of $[\mathrm{AH}, \mathrm{Theo}-$

5.1. Lemma. Suppose that $\alpha p>n-1$. Then there is $q_{0}>1$ such that

$$
\tau_{\alpha, p} \subset \tau_{n-1, q}
$$

for all $q \in\left(1, q_{0}\right)$.

Proof. We show that the assertion follows from [AH, Theorem B].

We may assume that $\alpha p<n$. Then we need only to show that there is $q>1$ such that

$$
\frac{(n-1)(p-1)+n}{p} \leq \frac{\alpha(q-1)+n}{q} .
$$

Since $p>1$, the left side of (5.2) is less than $n$. Hence there is $q_{0}>1$ such that (5.2) holds for $q \leq q_{0}$ because

$$
\frac{\alpha(q-1)+n}{q} \rightarrow n
$$

as $q \rightarrow 1$. The lemma is proved.

The next lemma is essential, see [HK3, 3.4], [LM, 3.16] and [MS] for special cases.

5.3. Lemma. Suppose that $\alpha p>n-1$. If $U$ is an $(\alpha, p)$-fine neighborhood of $x_{0}$, then there is a sequence of radii $r_{i} \rightarrow 0$ such that

$$
\partial B\left(x_{0}, r_{i}\right) \subset U \text {. }
$$

Proof. Write $B(r)=B\left(x_{0}, r\right)$ for $r>0$. In the light of Lemma 5.1 we may assume that $\alpha=n-1$ and that $\alpha p<n$. Let $E=U^{c}$. We may further assume that $E$ is open. Let $q=\frac{1}{2}(\alpha p+n-1)>n-1$ and write $h(r)=r^{n-q}, r>0$. Then

$$
I(r)=\int_{0}^{2 r}\left(\frac{h(t)}{t^{n-\alpha p}}\right)^{1 / p} \frac{d t}{t}=c(n, p) r^{(n-q) / p} r^{(\alpha p-n) / p}
$$

and hence it follows from Theorem 4.1 and Remark 4.4 that, for $r \leq 1$,

$$
\begin{aligned}
\frac{H_{h}(E \cap B(r))}{r^{n-q}} & \leq c \frac{\operatorname{cap}_{\alpha, p}(E \cap B(r), B(2 r))}{r^{n-\alpha p}} \\
& \leq c(n, p) \frac{B_{\alpha, p}(E \cap B(r))}{r^{n-\alpha p}} .
\end{aligned}
$$


Fix $r \leq \frac{1}{2}$ and suppose that for each $\rho \in\left[\frac{1}{2} r, r\right]$ the sphere $\partial B(\rho)$ meets $E$. Then it follows easily, cf. Remark 5.7 (b) below, that

$$
H_{h}(E \cap B(r)) \geq H_{h}\left(\left[\frac{1}{2} r e_{1}, r e_{1}\right]\right) \geq c(n, p) r^{n-q}
$$

where $e_{1}=(1,0, \ldots, 0)$. Then $(5.4)$ and (5.5) yield

$$
\frac{B_{\alpha, p}(E \cap B(r))}{r^{n-\alpha p}} \geq c(n, p)>0 .
$$

On the other hand, since $E$ is $(\alpha, p)$-thin at $x_{0}$ we can find an integer $j_{0}$ such that for $r=2^{-j}, j=j_{0}, j_{0}+1, \ldots$,

$$
\frac{B_{\alpha, p}(E \cap B(r))}{r^{n-\alpha p}}<c
$$

where $c$ is the constant of (5.6). This proves the lemma.

5.7. Remarks. (a) Lemma 5.3 fails to hold for $\alpha p \leq n-1$. In fact, the line segment $E=\left(0, e_{1}\right]$ is of $(\alpha, p)$-capacity zero [M1, Theorem 21] whence $E^{c}$ is an $(\alpha, p)$-fine neighborhood of 0 .

(b) To establish (5.5) above we made use of the following simple symmetrization property of Hausdorff measures: Let $E \subset \mathbf{R}^{n}$ and write $E^{*}=\left\{|x| e_{1}: x \in\right.$ $E\}$. Then $H_{h}\left(E^{*}\right) \leq H_{h}(E)$.

The main result of this section is

5.8. Theorem. Suppose that $\alpha p>n-1$ and that $U$ is an $(\alpha, p)$-finely open set. Then the following are equivalent.

(1) $U$ is $(\alpha, p)$-finely connected.

(2) $U$ is arcwise connected.

(3) $U$ is (euclidean) connected.

Proof. The implication of $(1) \Longrightarrow(2)$ was proved in [AL, Corollary 2] for all $\alpha p>1$. Since (2) trivially implies (3) we need only to show that (3) implies (1). This, in turn, immediately follows from [LMZ, 5.4] and the next lemma.

5.9. Lemma. Let $\alpha p>n-1$. If $V$ and $W$ are disjoint $(\alpha, p)$-finely open euclidean connected sets, then

$$
V \cap \bar{W}=\emptyset
$$

Proof. Suppose, on the contrary, that $x_{0} \in V \cap \bar{W}$. Let $x_{1} \in W$. In the light of Lemma 5.3 we can pick a radius $0<r<\left|x_{1}-x_{0}\right|$ such that

$$
\partial B\left(x_{0}, r\right) \subset V \subset W^{c} .
$$

Then let $x_{2} \in W \cap B\left(x_{0}, r\right)$. Since $\partial B\left(x_{0}, r\right)$ separates $x_{1}$ and $x_{2}$ in $W, W$ cannot be connected, and the lemma follows. 
5.10. Remarks. (a) Let $U$ be an $(\alpha, p)$-finely open and $(\alpha, p)$-finely connected set with $\alpha p>1$. It follows from [AL, Theorem 2] that each two points in $U$ can be joined by a coordinate path in $U$.

(b) Suppose that $\alpha p \leq n-1$. Then the statement (2) (and hence (3)) of Theorem 5.8 does not imply (1). To see this, suppose first that $\alpha p>1$. Let $E$ be the line segment $\left(0, e_{1}\right]$. Since $E$ is of $(\alpha, p)$-capacity zero we may choose an open connected neighborhood $D$ of $E$ such that $D$ is $(\alpha, p)$-thin at 0 . Thus $D^{c}$ is an $(\alpha, p)$-fine neighborhood of 0 . Let $V$ be the $(\alpha, p)$-fine component of the $(\alpha, p)$-fine interior of $D^{c}$ containing 0 . Then $V$ is $(\alpha, p)$-finely open and arcwise connected by Theorem 3.15 and [AL, Corollary 2]. Thus

$$
U=D \cup V
$$

is $(\alpha, p)$-finely open and arcwise connected. However, $U$ is not $(\alpha, p)$-finely connected.

Next, using the inclusion relations among fine topologies $[\mathrm{AH}$, Theorem $\mathrm{B}]$ we obtain a counterexample also for $\alpha p \leq 1$ if $n \geq 3$. The plane case follows from a slightly more careful but similar reasoning.

(c) For $\alpha p \leq 1$ the implication of (1) $\Longrightarrow(2)$ in Theorem 5.8 is false as shown in [AL, p. 62], cf. Remark 3.13.

(d) Theorem 5.8 is known in the plane for the classical fine topology, cf. [F3, Theorem 3] and [GL].

\section{Asymptotic paths for $\mathcal{A}$-subharmonic functions}

In this final section we give an application of Theorem 5.8. We show that there is a coordinate path along which an entire $\mathcal{A}$-subharmonic function which is not bounded from above tends to infinity. However, due to Theorem 5.8, we are confined to the case $p>n-1$.

Recall that the $(1, p)$-fine topology, $1<p \leq n$, is intimately connected to the (nonlinear) potential theory of $\mathcal{A}$-subharmonic functions. More precisely, let $\mathcal{A}: \mathbf{R}^{n} \times \mathbf{R}^{n} \rightarrow \mathbf{R}^{n}$ be a mapping which satisfies the usual assumptions of measurability, boundedness, ellipticity, coercivity, and homogeneity (that is, the assumptions $(2.1)-(2.5)$ in $[\mathrm{HKM}]$ or in $[\mathrm{H}])$. Continuous weak solutions to the equation (1.3) are called $\mathcal{A}$-harmonic, and an upper semicontinuous function $u$ in an open set $\Omega$ is termed $\mathcal{A}$-subharmonic if for each domain $D$, compactly contained in $\Omega$, and each $\mathcal{A}$-harmonic $h \in C(\bar{D}), h \geq u$ in $\partial D$ implies $h \geq u$ in $D$.

For basic properties of $\mathcal{A}$-subharmonic functions and their potential theory we refer to [HK 1-3], [K].

It was proved in $[\mathrm{H}]$ that if $u$ is an entire $\mathcal{A}$-subharmonic function in $\mathbf{R}^{n}$, and not bounded above, then there is a path $\Gamma, \Gamma(t) \rightarrow \infty$ as $t \rightarrow \infty$, such that $u(x) \rightarrow \infty$ as $x$ tends to $\infty$ along $\Gamma$. In the classical theory of subharmonic 
functions it is known that the path $\Gamma$ can be chosen to be polygonal; this was first proved by L. Carleson. We refer to [F3] for a lucid survey on the subject.

We do not know whether a polygonal path can always be found for general $\mathcal{A}$-subharmonic functions. However, we apply Theorem 5.8 and show that this is the case at least for $p>n-1$.

6.1. Theorem. Suppose that $p>n-1$ and that $u$ is $\mathcal{A}$-subharmonic in $\mathbf{R}^{n}$, unbounded from above. Then there is a coordinate path $\Gamma$ going to infinity such that

$$
\lim _{\substack{x \rightarrow \infty \\ x \in \Gamma}} u(x)=\infty
$$

By a coordinate path we mean a path which is a countable union of (possibly degenerated) line segments parallel to the coordinate axes.

Before we indicate how Theorem 5.8 can be used to deduce Theorem 6.1 some remarks about $(1, p)$-fine topology and $\mathcal{A}$-subharmonic functions are due.

In $[\mathrm{HKM}]$ the $\mathcal{A}$-fine topology $\tau_{\mathcal{A}}$ was defined to be the coarsest topology in $\mathbf{R}^{n}$ making all $\mathcal{A}$-subharmonic functions in $\mathbf{R}^{n}$ continuous. It was then shown in $[\mathrm{HKM}]$ that

$$
\tau_{\mathcal{A}}=\tau_{1, p}
$$

In effect, in $[\mathrm{HKM}]$ a seemingly different Wiener criterion was used, namely

$$
\int_{0}^{1}\left(r^{p-n} \operatorname{cap}_{1, p}\left(E \cap B\left(x_{0}, r\right), B\left(x_{0}, 2 r\right)\right)\right)^{1 /(p-1)} \frac{d r}{r}<\infty .
$$

However, this integral converges simultaneously with the integral in (1.1). For $p<n$ this is an immediate consequence of (4.6) and for completeness we provide a proof in the case $p=n$ (the fact that the two Wiener criteria coincide also when $p=n$ is evidently part of the folklore). Thus, let $p=n$ and define the capacity

$$
C_{1, n}(E)=\inf \left\{\int_{\mathbf{R}^{n}}\left(|\nabla v|^{n}+|v|^{n}\right) d m: v \in C_{0}^{\infty}\left(\mathbf{R}^{n}\right), v \geq 1 \text { in } E\right\} .
$$

Then the $C_{1, n}$-capacity is equivalent to the $B_{1, n}$-capacity, cf. [AM] or [Hed]. We prove

6.3. Proposition. Let $E \subset \mathbf{R}^{n}$ and $x \in \mathbf{R}^{n}$. Then

$$
\sum_{k=1}^{\infty}\left(\operatorname{cap}_{1, n}\left(E \cap B_{k}, B_{k-1}\right)\right)^{1 /(n-1)}<\infty
$$

if and only if

$$
\sum_{k=1}^{\infty}\left(C_{1, n}\left(E \cap B_{k}\right)\right)^{1 /(n-1)}<\infty
$$

Here $B_{k}=B\left(x, 2^{-k}\right)$. 
Proof. The "only if" part being a trivial consequence of Poincaré's inequality, we only prove the converse. For $k \in \mathrm{N}$ let $u_{k}$ be the $C_{1, n}$-capacitary potential of $E \cap B_{k}$. Let $d_{k}=\sup _{\partial B_{k-1}} u_{k}$. We show that $\lim _{k \rightarrow \infty} d_{k}=0$. This will complete the proof since then for $k$ big enough $d_{k}<\frac{1}{2}$, and it follows that

$$
C_{1, n}\left(E \cap B_{k}\right) \geq 2^{-n} \operatorname{cap}_{1, n}\left(E \cap B_{k}, B_{k-1}\right)
$$

which implies the assertion.

To this end, suppose that $d=\limsup d_{k}>0$. Then using the Harnack inequality for $u_{k}$ outside $\bar{B}_{k}$ and the minimum principle (see [S]) we obtain an infinite set $I \subset \mathbf{N}$ such that

$$
\inf _{\bar{B}_{k-1}} u_{k} \geq c(n) d
$$

for every $k \in I$.

Write

$$
S=\bigcup_{k \in I} \partial B_{k-1}
$$

Fix $k \in \mathbf{N}$ and pick the least $j \in I$ such that $j \geq k$. Then

$$
\begin{aligned}
C_{1, n}\left(E \cap B_{k}\right) & \geq C_{1, n}\left(E \cap B_{j}\right) \geq(c d)^{n} C_{1, n}\left(B_{j-1}\right) \\
& \geq(c d)^{n} C_{1, n}\left(S \cap B_{k}\right) .
\end{aligned}
$$

Then the set $S$ is $(1, n)$-thin at $x$, contradicting Lemma 3.12. Thus $d=0$ and the proposition is proved.

Proof of Theorem 6.1. Let $u$ be an $\mathcal{A}$-subharmonic function in $\mathbf{R}^{n}$, unbounded from above. Then there is a number $L_{0}>0$ such that for each $L>L_{0}$ $u$ is unbounded in the set $K(x, L)$, where $K(x, L)$ is the union of all continua which contain $x$ and on which $u$ is $\geq L$, see $[\mathrm{H}, 4.5]$. Pick points $x_{j}$ in $\mathbf{R}^{n}$ inductively as follows. Let $x_{1}$ be any point such that $u\left(x_{1}\right)>2 L_{0}$, and suppose that $x_{1}, \ldots, x_{j}$ have been chosen. Let $x_{j+1}$ be a point in $K\left(x_{j}, u\left(x_{j}\right)\right)$ such that $u\left(x_{j+1}\right)>2^{j+1} L_{0}$. Then $x_{j}$ and $x_{j+1}$ can be joined by a continuum in the finely open set $\left\{u>2^{j} L_{0}\right\}$. Since $p>n-1$, Theorem 5.8 implies that $x_{j}$ and $x_{j+1}$ belong to the same fine component $U_{j}$ of $\left\{u>2^{j} L_{0}\right\}$; in particular, there is a coordinate path $\Gamma_{j}$ joining $x_{j}$ and $x_{j+1}$ in $U_{j}$ [AL, Theorem 2]. Then $\Gamma=\cup \Gamma_{j}$ is the required path, and the theorem is proved. 


\section{References}

[AH] AdAms, D.R., and L.I. HEDBERG: Inclusion relations among fine topologies in non-linear potential theory. - Indiana Univ. Math. J. 33, 1984, 117-126.

[AL] Adams, D.R., and J.L. LEwIS: Fine and quasi connectedness in nonlinear potential theory. - Ann. Inst. Fourier (Grenoble) 35.1, 1985, 57-73.

[AM] Adams, D.R., and N.G. Meyers: Thinness and Wiener criteria for non-linear potentials. - Indiana Univ. Math. J. 22, 1972, 169-197.

[D] Dоов, J.L.: Classical potential theory and its probabilistic counterpart. - Springer-Verlag, New York-Berlin-Heidelberg-Tokyo, 1984.

[F1] Fuglede,B.: The quasi topology associated with a countable subadditive set function. Ann. Inst. Fourier (Grenoble) 21.1, 1971, 123-169.

[F2] Fuglede, B.: Connexion en topologie fine et balayage des mesures. - Ann. Inst. Fourier (Grenoble) 21.3, 1971, 227-244.

[F3] FUGLEDE, B.: Asymptotic paths for subharmonic functions and polygonal connectedness of fine domains. - Séminaire de Theorie du Potentiel, Paris, No. 5. Lecture Notes in Mathematics 814. Springer-Verlag, New York-Berlin-Heidelberg, 1980, 97-116.

[GL] Gamelin, T.W., and T.J. Lyons: Jensen measures for $R(K)$. - J. London Math. Soc. $27,1983,317-330$.

[Hed] Hedberg, L.I.: Nonlinear potential theory and Sobolev spaces. - Nonlinear analysis, function spaces and applications, Vol. 3 (Proceedings of the Spring School held in Litomyšl, 1986). Teubner-Verlag (to appear).

[HW] Hedberg, L.I., and Th.H. WolfF: Thin sets in nonlinear potential theory. - Ann. Inst. Fourier (Grenoble) 33.4, 1983, 161-187.

[H] Heinonen, J.: Asymptotic paths for subsolutions of quasilinear elliptic equations. - Manuscripta Math. 62, 1988, 449-465.

[HK1] Heinonen, J., and T. KilpeläInen: $\mathcal{A}$-superharmonic functions and supersolutions of degenerate elliptic equations. - Ark. Mat. 26, 1988, 87-105.

[HK2] Heinonen, J., and T. KilpeläInen: Polar sets for supersolutions of degenerate elliptic equations. - Math. Scand. 63, 1988, 136-150.

[HK3] Heinonen, J., and T. KILPELÄInEN: On the Wiener criterion and quasilinear obstacle problems. - Trans. Amer. Math. Soc. 310, 1988, 239-255.

[HKM] Heinonen, J., T. KilpeläInen, and O. Martio: Fine topology and quasilinear elliptic equations. - Ann. Inst. Fourier (Grenoble) 39.2, 1989, 293-318.

[K] KILPELÄINEN, T.: Potential theory for supersolutions of degenerate elliptic equations. Indiana Univ. Math. J. 38, 1989, 253-275.

[LM] Lindqvist, P., and O. Martio: Two theorems of N. Wiener for solutions of quasilinear elliptic equations. - Acta Math. 155, 1985, 153-171.

[LMZ] LuKEš, J., J. MALÝ, and L. ZAJÍČEK: Fine topology methods in real analysis and potential theory. - Lecture Notes in Mathematics 1189. Springer-Verlag, New York-BerlinHeidelberg-Tokyo, 1986.

[Mar] Martio, O.: Capacity and measure densities. - Ann. Acad. Sci. Fenn. Ser. A I Math. 4, 1978/1979, 109-118.

[MS] Martio, O., and J. Sarvas: Density conditions in the $n$-capacity. - Indiana Univ. Math. J. 26, 1977, 761-776.

[Maz] MaZ'Ya, V.G.: Sobolev spaces. - Springer-Verlag, Berlin-Heidelberg-New York-Tokyo, 1985. 
[MK] MaZ'YA, V.G., and V.P. Khavin: Non-linear potential theory. - Russ. Math. Surveys $27,1972,71-148$.

[M1] MEYers, N.G.: A theory of capacities for potentials of functions in Lebesgue classes. Math. Scand. 26, 1970, 255-292.

[M2] Meyers, N.G.: Continuity properties of potentials. - Duke Math. J. 42, 1975, 157-166.

[R1] Reshetnyak, Yu.G.: Space mappings with bounded distortion. - Sibirsk. Mat. Zh. 8, 1967, 629-658 (Russian).

[R2] REshetNYAK, YU.G.: The concept of capacity in the theory of functions with generalized derivatives. - Sibirsk. Mat. Zh. 10, 1969, 1109-1138 (Russian).

[S] SERrin, J.: Local behavior of solutions of quasi-linear equations. - Acta Math. 111, 1964, 247-302.

[St] Stein, E.M.: Singular integrals and differentiability properties of functions. - Princeton University Press, Princeton, 1970.

University of Michigan

Department of Mathematics

Ann Arbor, MI 48109

U.S.A. (current address)

University of Jyväskylä

Department of Mathematics

Seminaarinkatu 15

SF-40100 Jyväskylä

Finland

Received 3 April 1989
University of Jyväskylä

Department of Mathematics

Seminaarinkatu 15

SF-40100 Jyväskylä

Finland
Charles University

Department of Mathematical Analysis

Sokolovská 83

18600 Praha 8

Czechoslovakia 